\title{
Critical decay exponent of the pair contact process with diffusion
}

\author{
$\mathrm{Su}-$ Chan Park (박수 찬) \\ Department of Physics, The Catholic University of Korea, Bucheon 420-743, Republic of Korea
}

(Dated: November 21, 2018)

\begin{abstract}
We investigate the one-dimensional pair contact process with diffusion (PCPD) by extensive Monte Carlo simulations, mainly focusing on the critical density decay exponent $\delta$. To obtain an accurate estimate of $\delta$, we first find the strength of corrections to scaling using the recently introduced method [S.-C. Park. J. Korean Phys. Soc. 62, 469 (2013)]. For small diffusion rate $(d \leq 0.5)$, the leading corrections-to-scaling term is found to be $\sim t^{-0.15}$, whereas for large diffusion rate $(d=0.95)$ it is found to be $\sim t^{-0.5}$. After finding the strength of corrections to scaling, effective exponents are systematically analyzed to conclude that the value of critical decay exponent $\delta$ is $0.173(3)$ irrespective of $d$. This value should be compared with the critical decay exponent of the directed percolation, 0.1595 . In addition, we study two types of crossover. At $d=0$, the phase boundary is discontinuous and the crossover from the pair contact process to the PCPD is found to be described by the crossover exponent $\phi=2.6(1)$. We claim that the discontinuity of the phase boundary cannot be consistent with the theoretical argument supporting the hypothesis that the PCPD should belong to the DP. At $d=1$, the crossover from the mean field PCPD to the PCPD is described by $\phi=2$ which is argued to be exact.
\end{abstract}

PACS numbers: 05.70.Ln, 05.70.Jk, 64.60.Ht

\section{INTRODUCTION}

The pair contact process with diffusion (PCPD) is an interacting particle system with diffusion, pair annihilation $(2 A \rightarrow 0)$, and branching by pairs $(2 A \rightarrow 3 A)$. The PCPD was introduced in 1982 by Grassberger [1], but had remained unnoticed in the statistical physics community for about 15 years. Since Howard and Täuber 2] (re)introduced the so-called 'bosonic' PCPD in 1997, the PCPD has been captivating statistical physicists and the effort to understand the critical behavior of the PCPD has continued until now [3-19].

It was accepted almost without question that the upper critical dimension of the PCPD is 2 and the PCPD does not belong to the directed percolation (DP) universality class in higher dimensions [20]. On the other hand, the one dimensional PCPD has gained its notorious fame from the beginning because of its strong corrections to scaling. Consequently, numerical studies have reported scattered values of critical exponents (see Table I of Ref. [16] for a summary of reported values of critical exponents) and, in turn, as many hypotheses concerning the critical behavior were suggested as the number of research groups involved (for a review of the early various scenarios, see Ref. [21]). It still remains an open question whether the one dimensional PCPD belongs to the DP class (DP hypothesis) or forms a different universality class just like the higher dimensional PCPD (non-DP hypothesis). Since we are mainly interested in the PCPD in one dimension, by the PCPD in the following we will exclusively mean the one-dimensional PCPD, unless dimensions are explicitly stated.

To support the DP hypothesis, Hinrichsen [22] provided a theoretical argument as to why the PCPD should belong to the DP class. This argument begins with the numerical observation that the dynamic exponent of the PCPD is smaller than 2, which means critical clusters spread super-diffusively. Since isolated particles can spread at most diffusively with dynamic exponent 2 , diffusive motion of isolated particles can be regarded as frozen in comparison to the critical spreading and, in turn, the effectively frozen isolated particles can at best play the role of isolated particles of the pair contact process (PCP) without diffusion which is known to belong to the DP class. Accordingly, the PCPD should belong to the DP class. Although this argument is not unquestionable (see Ref. 23] for a critique), it is quite persuasive once the dynamic exponent of the PCPD is accepted to be smaller than 2 as numerical studies suggest. We will discuss this argument at the end of Sec. IV A Barkema and his colleagues have reported numerical results to support the DP hypothesis [7, 16, 18].

At the same time, numerical studies supporting the non-DP hypothesis are also available. The critical behavior of the driven pair contact process with diffusion [11, 12] seems to suggest that the PCPD cannot be described by a field theory with a single component field, which makes the PCPD not satisfy the prerequisite of the DP conjecture [1, 24]. Besides, the existence of non-trivial crossover from the PCPD to the DP [14, 17] was invoked to support the non-DP nature of the PCPD.

Since the controversy arises from numerical difficulty of finding accurate value of critical exponents due to strong corrections to scaling, it is necessary to tame the corrections to scaling at our disposal. To this end, this paper exploits a systematic method suggested in Ref. 25] to find corrections to scaling without prior knowledge of leading asymptotic behavior. Once the strength of corrections to scaling is determined, the effective exponent can be systematically analyzed to get an accurate estimate of the critical exponent. In this paper, we find the critical decay exponent $\delta$ by extensive Monte Carlo sim- 
ulations, using the method briefly mentioned above.

The paper is organized as follows: Section[I]consists of two parts. To be self-contained, Sec. II introduces the dynamic rules of the PCPD and describes the expected behavior of the order parameter in each phase. Also an algorithm to simulate the stochastic dynamics is detailed with comparison to previous studies. In Sec. II B. a method to estimate the leading corrections-to-scaling term is explained. Numerical estimate of the critical decay exponent is presented in Sec. III, using the method explained in Sec. IIB Section[V]studies crossover behavior from two extreme points of the model, $d=0$ (without diffusion) and $d \rightarrow 1$ with a suitable time rescale (mean field), to the PCPD. Section $\nabla$ summarizes the work.

\section{MODEL AND METHOD}

\section{A. Model}

The PCPD is defined on a one-dimensional lattice of size $L$ with periodic boundary conditions. Each site is either occupied by a particle $(A)$ or empty $(\varnothing)$ and every site can accommodate at most one particle. The dynamics of the PCPD are defined by the following transition events,

$$
\begin{gathered}
A \varnothing \leftrightarrow \varnothing A, \text { with rate } d, \\
A A \rightarrow \varnothing \varnothing, \text { with rate } p(1-d), \\
\varnothing A A, A A \varnothing \rightarrow A A A, \text { with rate }(1-p)(1-d) / 2,(1)
\end{gathered}
$$

where $0 \leq d<1$ and $0 \leq p \leq 1$. For bookkeeping purposes, we introduce a stochastic process $A_{n}(t)$ at every site $n$ which takes 1 (0) if site $n$ is occupied (vacant) at time $t$. We define the particle density, $\rho(t)$, the pair density, $\rho_{p}(t)$, and the triplet density, $\rho_{t}(t)$, as

$$
\begin{aligned}
\rho(t) & =\lim _{L \rightarrow \infty} \frac{1}{L} \sum_{n=1}^{L}\left\langle A_{n}(t)\right\rangle, \\
\rho_{p}(t) & =\lim _{L \rightarrow \infty} \frac{1}{L} \sum_{n=1}^{L}\left\langle A_{n}(t) A_{n+1}(t)\right\rangle, \\
\rho_{t}(t) & =\lim _{L \rightarrow \infty} \frac{1}{L} \sum_{n=1}^{L}\left\langle A_{n}(t) A_{n+1}(t) A_{n+2}(t)\right\rangle,
\end{aligned}
$$

where $\langle\ldots\rangle$ means the average over ensembles. The evolution equation of $\rho(t)$ is

$$
\frac{1}{1-d} \frac{d \rho(t)}{d t}=(1-3 p) \rho_{p}(t)-(1-p) \rho_{t}(t)
$$

When $p=p_{c}$ (critical point), both $\rho(t)$ and $\rho_{p}(t)$ are expected to show asymptotic power-law behavior with corrections to scaling as

$$
\begin{aligned}
\rho(t) & \sim a t^{-\delta}\left(1+c t^{-\chi}+o\left(t^{-\chi}\right)\right), \\
\rho_{p}(t) & \sim a^{\prime} t^{-\delta^{\prime}}\left(1+c^{\prime} t^{-\chi^{\prime}}+o\left(t^{-\chi^{\prime}}\right)\right),
\end{aligned}
$$

where $a, a^{\prime}, c, c^{\prime}$ are constants and $o\left(t^{-\chi}\right)$ and $o\left(t^{-\chi^{\prime}}\right)$ contain higher order terms which decay faster than $t^{-\chi}$ and $t^{-\chi^{\prime}}$, respectively. In one dimension, it is believed that $\delta^{\prime}$ equals $\delta$, whereas the mean field theory assumes $\delta^{\prime}=2 \delta$; see Sec. IV. Also it is believed that $\chi=\chi^{\prime}$, numerical evidence of which will be provided in Sec. III. On this account, we will drop the primes in the symbols of exponents for $\rho_{p}(t)$ in what follows and we will refer to $\delta$ and $\chi$ as the critical decay exponent and the leading corrections-to-scaling exponent (LCSE), respectively. In the active phase $\left(p<p_{c}\right.$ within our model definition), both $\rho(t)$ and $\rho_{p}(t)$ approach certain nonzero values exponentially as $t \rightarrow \infty$ and in the absorbing phase $\left(p>p_{c}\right)$, $\rho(t)$ and $\rho_{p}(t)$ decrease to zero faster than $t^{-\delta}$ for nonzero $d$.

There are two important limiting cases. When $d=$ 0, this model corresponds to the PCP [26] which has infinitely many absorbing states and belongs to the DP class. Meanwhile, taking $d \rightarrow 1$ limit with $\tau \equiv(1-d) t$ kept finite, the (site) mean field theory becomes exact. Hence there are two crossover behaviors at $d=0$ (from the PCP to the PCPD) and at $d=1$ (from the mean field PCPD to the PCPD). In Sec. IV we will study these two kinds of crossover behavior.

To simulate the model, we employ the following algorithm. At first, we introduce

$$
d t \equiv 1 / \max (2 d, 1-d)
$$

which makes $2 d d t$ and $(1-d) d t$ interpreted as probability. Now assume that there are $N(t)$ particles at time $t$. We randomly choose one particle among $N(t)$ particles with equal probability and choose one of two nearest neighbors of the chosen particle with equal probability. Let us assume that the chosen particle is located at site $n$ and the selected neighbor site is $n+k(k= \pm 1)$. If $A_{n+k}(t)=0$, the particle at site $n$ moves to site $n+k$ with probability $2 d d t$, but with probability $1-2 d d t$, nothing happens. In the case $A_{n+k}(t)=1$, two particles at sites $n$ and $n+k$ are removed with probability $p(1-d) d t$ (pair annihilation), the site $n+2 k$ becomes occupied with probability $(1-p)(1-d) d t$ (branching), or with probability $1-(1-d) d t$ nothing happens. If $A_{n+2 k}(t)$ is already 1 in the branching attempt, no change in the configuration happens. After the above attempt, time increases by $d t / N(t)$.

Notice that the PCPD studied in Refs. [11, 14] corresponds to the case with $d=\frac{1}{3}$ up to a time-rescale factor (time $t$ of the PCPD in Refs. [11, 14] corresponds to $\frac{3}{2} t$ of the case with $d=\frac{1}{3}$ in this paper). Also note that the simulation algorithm employed in Ref. [27] is slightly different from that used in this paper. But if we set $d=D /(2-D)$ where $D$ is the diffusion parameter used for simulations in Ref. [27] and if we rescale time appropriately, the simulation results in Ref. [27] can be directly compared to those obtained by the algorithm in the above. For example, the reported critical point $\approx 0.13353$ of the case with $D=0.5$ in Ref. [27] is consistent with that in Ref. [14] which is $\approx 0.133519$. 


\section{B. Corrections to scaling}

A systematic way to find the critical decay exponent simultaneously together with the critical point is to investigate the behavior of the effective exponent $-\delta_{\text {eff }}(t)$ defined as $(b>1)$

$$
-\delta_{\mathrm{eff}}(t) \equiv \frac{\ln [\rho(t) / \rho(t / b)]}{\ln b},
$$

which, by Eq. (4), is expected to behave at the critical point as

$$
-\delta_{\mathrm{eff}}(t)=-\delta-c \frac{b^{\chi}-1}{\ln b} t^{-\chi}+o\left(t^{-\chi}\right) .
$$

In the time regime where higher order terms $o\left(t^{-\chi}\right)$ are negligibly small but the leading correction term $t^{-\chi}$ is not negligible, a plot of the effective exponent against $t^{-\chi}$ with the correct value of $\chi$ should be a straight line if the system is at the critical point. Meanwhile, it veers up (down) if the system is in the active (absorbing) phase as $t^{-\chi}$ gets smaller. From the expected behavior in each phase, the critical exponent and the critical point can be found simultaneously by investigating the behavior of effective exponents near criticality, once the LCSE $\chi$ is known. Hence the information about the LCSE is indispensable in order to estimate the critical decay exponent and the critical point accurately via the effective exponents.

A systematic method to find corrections to scaling without knowing $\delta$ was recently suggested [25]. The idea is that at the critical point the double ratio of $\rho$ at three different time points should behave asymptotically as

$$
\frac{\rho(t)}{\rho\left(t / b_{1}\right)} / \frac{\rho\left(t / b_{1}\right)}{\rho\left(t / b_{1}^{2}\right)}=1+c\left(b_{1}^{\chi}-1\right)^{2} t^{-\chi}+o\left(t^{-\chi}\right),
$$

where $b_{1}$ is a (fixed) constant. Although $b_{1}$ is not necessarily the same as $b$ in Eq. (8), we will use the same value for $b$ and $b_{1}$ in this paper and we will drop the subscript in $b_{1}$ in the following. We introduce a corrections-to-scaling function (CTSF) $\Theta(t)$ as the logarithm of the left hand side of Eq. (9),

$$
\Theta(t)=\ln \rho(t)+\ln \rho\left(t / b^{2}\right)-2 \ln \rho(t / b),
$$

which behaves at criticality as

$$
\Theta(t) \sim c\left(b^{\chi}-1\right)^{2} t^{-\chi}+o\left(t^{-\chi}\right) .
$$

The behavior of $\Theta(t)$ at off-criticality is also of interest. If the system is slightly away from the critical point with $p=p_{c}+\Delta p, \Theta(t)$ is indistinguishable from Eq. (11) up to $t \sim|\Delta p|^{-\nu_{\|}}$, where $\nu_{\|}$is the critical exponent describing the divergence of correlation time. Since $\Theta(t)$ can be understood as a curvature of the curve $\ln \rho(t)$ as a function of $\ln t$, that is, $\Theta(t) \approx \frac{d^{2} \ln \rho(t)}{d \ln t^{2}}, \Theta(t)$ gets larger (smaller) with $t$ if the system is in the active (absorbing) phase. Hence, if the coefficient of $c$ in Eq. (11) is positive,

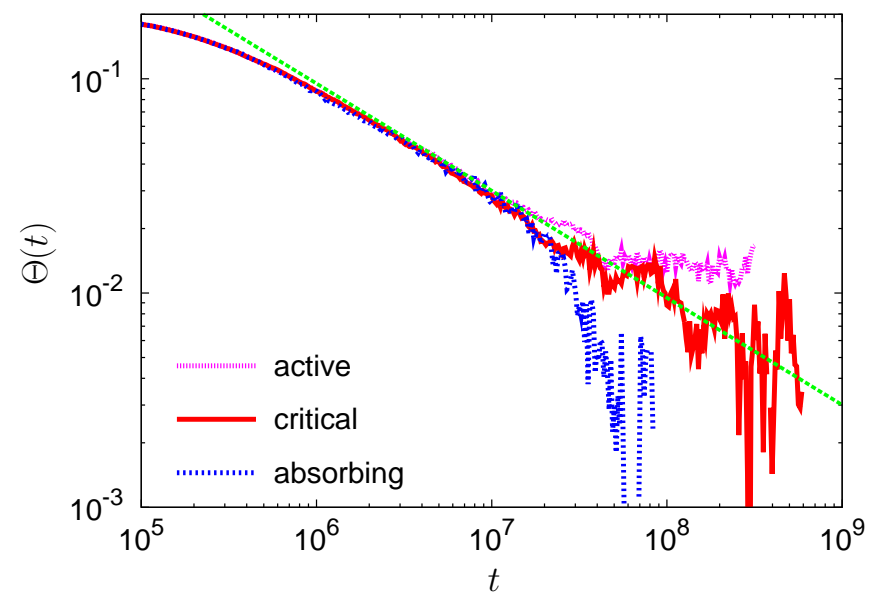

FIG. 1. (Color online) Double logarithmic plots of $\Theta(t)$ vs $t$ around the critical point of the PCPD with $d=0.95$. The curves correspond to $p=0.258110$ (active), 0.258112 (critical), and 0.258114 (absorbing) from top to bottom; see Sec [II. The straight line with slope -0.5 is for guides to the eyes.

a typical behavior of $\Theta(t)$ around the critical point looks like Fig. 1. These curves are obtained from simulations of the PCPD with $d=0.95$. To be specific, the system size is $L=2^{21}$ and the number of independent runs are 9800 , 7200 , and 1600 for $p=0.258110$ (active), 0.258112 (critical), and 0.258114 (absorbing), respectively. Due to the double derivative-like nature of $\Theta(t)$, the curves obtained from numerical simulations can be very noisy as seen in Fig. 1] unless the number of independent simulation runs is very large. In this respect, $\Theta(t)$ alone may not be a good measure to find the critical point and the analysis of the effective exponents which are less noisy than $\Theta(t)$ should be accompanied.

Although the behavior of $\Theta(t)$ looks qualitatively similar to $\rho(t)$ around the critical point, $\Theta(t)$ in the active phase actually should approach zero as $t \rightarrow \infty$ because $\rho(t)$ in this limit saturates to a finite number with zero curvature. We will soon see such a long time behavior in the active phase from an exactly solvable model. In the absorbing phase, the behavior of $\Theta(t)$ in the limit of infinite time depends on the asymptotic behavior of $\rho(t)$. If $\rho(t)$ decreases exponentially, so does $\Theta(t)$. On the other hand, if $\rho(t)$ decreases as a power-law in the absorbing phase like the PCPD, $\Theta(t)$ should also approach zero as $t \rightarrow \infty$. Even though $\Theta(t)$ of the PCPD should approach zero in all phases as $t \rightarrow \infty$, the deviation of $\Theta(t)$ at some point from the critical $\Theta(t)$ is conspicuous as Fig. 1 illustrates. Thus, such infinite time behavior does not limit the practical usefulness.

The sign of $c$ is not necessarily positive and an example of the case with a negative $c$ can be found by the following equation

$$
\frac{d \rho(t)}{d t}=r \rho(t)-\rho(t)^{2}
$$




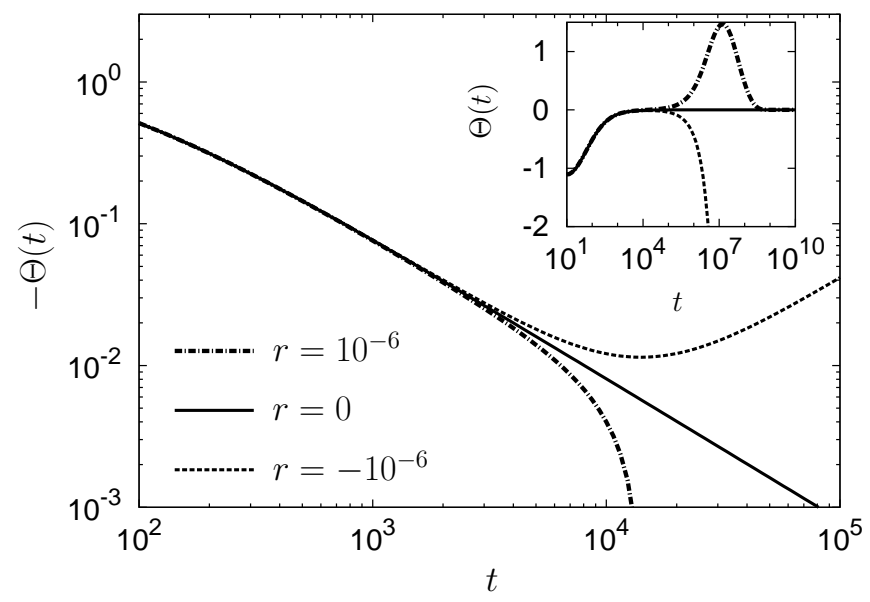

FIG. 2. Log-log plots of $-\Theta(t)$ vs $t$ around the critical point of the model Eq. (12). $\Theta(t)$ is calculated using Eq. (13) with $b=10$. Inset: Semi-logarithmic plots of $\Theta(t)$ vs $t$ for the same values of $r$ 's as in the main figure. While $\Theta(t)$ for negative $r$ (in the absorbing phase) decreases exponentially, $\Theta(t)$ for positive $r$ (in the active phase) approaches 0 as $t \rightarrow \infty$.

with initial condition $\rho(t=0)=1$. The solution is

$$
\rho(t)= \begin{cases}\left(e^{-r t}+\left(1-e^{-r t}\right) / r\right)^{-1}, & r \neq 0, \\ (1+t)^{-1} \sim t^{-1}\left(1-t^{-1}\right), & r=0 .\end{cases}
$$

Since the coefficient of the leading corrections-to-scaling term at the critical point $(r=0)$ is negative, it is appropriate to draw $-\Theta(t)$ vs $t$ on a double logarithmic scale as in Fig. 2 which makes the curve in the active (absorbing) phase veer down (up) contrary to Fig. 1. The inset of Fig. 2 shows that $\Theta(t)$ in the active phase approach 0 as $t \rightarrow \infty$ as argued before. Since $\rho(t)$ decreases exponentially in the absorbing phase of this example, $\Theta(t)$ also decreases exponentially to $-\infty$.

\section{CRITICAL DECAY EXPONENT}

This section investigates the critical decay exponent $\delta$ of the PCPD for various $d$ 's via the analysis of the effective exponents along with the corresponding CTSFs. For all numerical analyses in this section, the system size is $L=2^{21}$ and the initial density is 1 . Since $\rho(t)$ and $\rho_{p}(t)$ are equally important, the CTSFs for both $\rho(t)$ and $\rho_{p}(t)$ are studied and will be denoted by $\Theta_{r}(t)$ and $\Theta_{p}(t)$, respectively.

At first, we will present the results for the case with $d=0.1$. As we will show later, the critical point is found to be $p_{c}=0.111158(1)$, where the number in parentheses indicates the uncertainty of the last digit. Figure 3 shows double-logarithmic plots of $\Theta_{r}$ and $\Theta_{p}$ vs $t$ at $p=0.111158$ for $b=10$. These data are obtained from 3000 independent runs at the designated value of $p$. It seems that $\Theta_{p}$ shows a power-law behavior as $\Theta_{p} \sim t^{-0.138}$ while $\Theta_{r}$ has no symptom of power-law

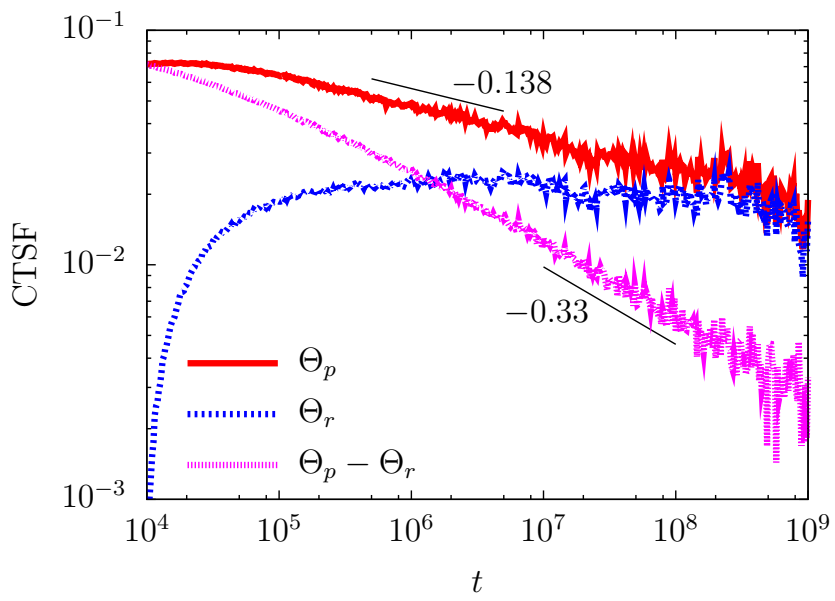

FIG. 3. (Color online) Log-log plots of CTSFs vs $t$ at $p=$ 0.111158 and $d=0.1$. Here, $b=10$ is used. Two line segments with slopes -0.138 and -0.33 as indicated in the figure are also drawn for guides to the eyes.

decay up to $t=10^{8}$. On the other hand, $\Theta_{r}$ and $\Theta_{p}$ become almost overlapped after $t=10^{8}$, which implies that not only the LCSE but also the coefficients of the leading correction-to-scaling terms of $\rho$ and $\rho_{p}$ are identical. Since $\Theta_{p}$ exhibits a more or less clean power-law behavior and $\Theta_{r}$ eventually follows $\Theta_{p}$, we estimate $\chi$ to be 0.138 from $\Theta_{p}$. Note that this estimate is comparable to that in Ref. [18].

It is worthwhile to discuss the implication of the difference between the two CTSFs, $\Theta_{p}-\Theta_{r}$, which shows a clean power-law behavior with $\simeq t^{-0.33}$ albeit $\Theta_{p} \sim$ $t^{-0.138}$. By definition of the CTSF, $\Theta_{p}-\Theta_{r}$ can be regarded as the CTSF for $\rho_{p}(t) / \rho(t)$. Hence if one analyzes $\rho_{p}(t) / \rho(t)$ rather than $\rho(t)$ and $\rho_{p}(t)$ separately with the assumption of $c \neq c^{\prime}$ in Eqs. (4) and (5), one may wrongfully conclude that the corrections to scaling of the PCPD are weaker than the actual strength $t^{-0.138}$. In fact, the cancellation of the leading corrections-to-scaling term in $\rho_{p}(t) / \rho(t)$ was already anticipated in Ref. [18] and we confirmed it through the direct numerical analysis.

Actually, the cancellation of the leading corrections-toscaling term in the ratio of two quantities is not unusual. An immediate example arises when we analyze Eq. (3). Since $\chi<1, \rho_{p}(t)$ and $\rho_{t}(t)$ at the critical point should be

$$
\begin{aligned}
& \rho_{p}(t)=a_{p} t^{-\delta}\left(1+c t^{-\chi}+\Xi(t)+e_{p} t^{-1}+o\left(t^{-1}\right)\right), \\
& \rho_{t}(t)=a_{t} t^{-\delta}\left(1+c t^{-\chi}+\Xi(t)+e_{t} t^{-1}+o\left(t^{-1}\right)\right),
\end{aligned}
$$

where $a_{p}$ and $a_{t}$ are constants satisfying $a_{t}\left(1-p_{c}\right)=$ $a_{p}\left(1-3 p_{c}\right), \Xi(t)$ contains all terms decaying faster than $t^{-\chi}$ but slower than $t^{-1}$, and $e_{p}$ should be strictly smaller than $e_{t}$. Otherwise, the leading power of the left hand side of Eq. (3) cannot be the same as that of the right hand side of the equation. Hence, $\rho_{p}(t) / \rho_{t}(t)$ at the crit- 


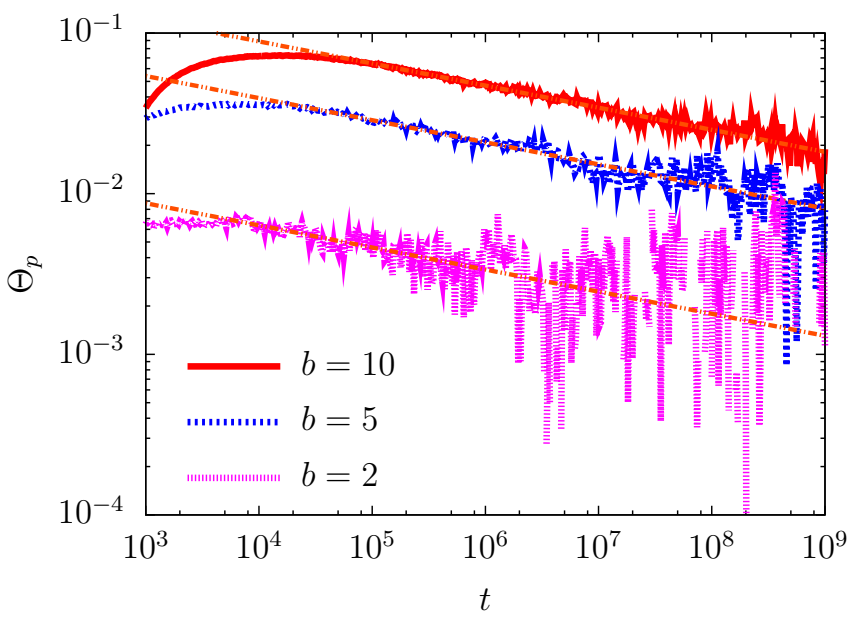

FIG. 4. (Color online) Plots of $\Theta_{p}$ vs $t$ at $p=0.111158$ and $d=0.1$ for $b=10,5$, and 2, from top to bottom. The straight line lying on $\Theta_{p}$ with $b=10$ is the result of the power-law fitting $c\left(b^{\chi}-1\right)^{2} t^{-\chi}$ with fitting parameters $c$ and $\chi$. Two straight lines lying on $\Theta_{p}$ 's for $b=5$ and $b=2$, respectively, are plots of Eq. (11) with $c$ and $\chi$ obtained from the fitting.

ical point should have the form

$$
\frac{\rho_{p}(t)}{\rho_{t}(t)}=\frac{1-p_{c}}{1-3 p_{c}}\left(1+\left(e_{p}-e_{t}\right) t^{-1}+o\left(t^{-1}\right)\right) .
$$

Unlike the cancellation of the leading corrections-toscaling terms in $\rho_{p} / \rho_{t}$, however, we could not find any theoretical reason why $\rho(t)$ and $\rho_{p}(t)$ should have exactly the same leading corrections-to-scaling term. This can be a theoretical challenge of the PCPD.

To check the consistency of Eq. (11), we analyze $\Theta_{p}$ 's for various values of $b(b=5$ and $b=2)$. We first fit $\Theta_{p}$ for $b=10$ using a fitting function $c\left(b^{\chi}-1\right)^{2} t^{-\chi}$ with two fitting parameters $c$ and $\chi$. From the fitting, we estimate $\chi \approx 0.138$ and the coefficient of the leading correctionsto-scaling term to be $c \approx 2.27$. The straight line lying on $\Theta_{p}$ for $b=10$ (top curve) in Fig. 4 is the result of this fitting. Then, we compare $c\left(b^{\chi}-1\right)^{2} t^{-\chi}$ for $b=5$ and $b=2$ with the estimated values of $c$ and $\chi$ to $\Theta_{p}$ 's for corresponding $b$ 's, which shows an excellent coincidence.

We think this coincidence provides a numerical evidence for the absence of logarithmic corrections in the leading corrections-to-scaling term. When we derive Eq. (11), we tacitly assumed that the leading term has no logarithmic corrections. If there happens to be such corrections, the above procedure should exhibit a systematic deviation for different values of $b$. Also a nice power-law behavior of $\Theta_{p}$ for about four decades, as seen in Fig. 4 suggests that logarithmic corrections, even if exist, are negligible. Furthermore, the clean power-law behavior of $\Theta_{p}-\Theta_{r}$ also provides an indirect evidence against logarithmic corrections.

Finding the LCSE to be 0.138 , we now analyze the effective exponent $-\delta_{\text {eff. }}$. Since $\Theta_{p}$ shows a cleaner powerlaw behavior than $\Theta_{r}$, we analyze $-\delta_{\text {eff }}$ calculated from

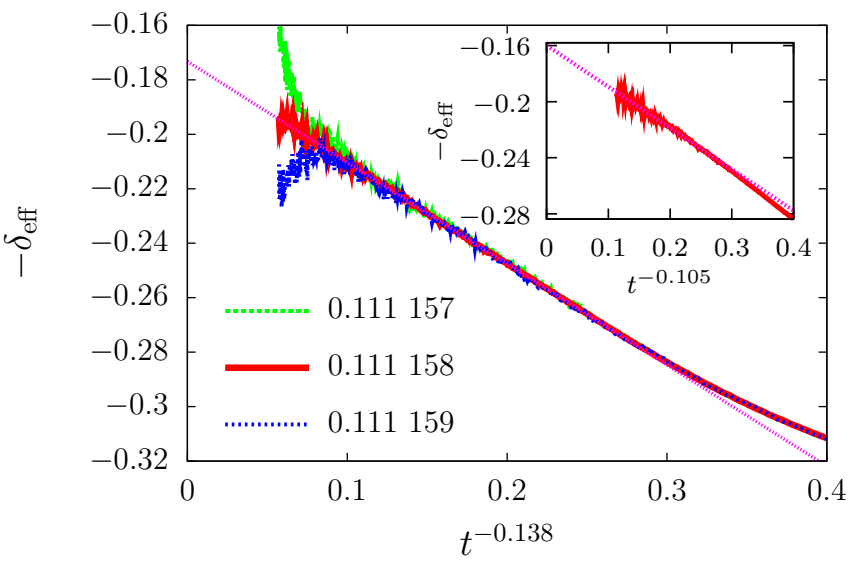

FIG. 5. (Color online) Plots of $-\delta_{\text {eff }}$ vs $t^{-0.138}$ for $p=$ $0.111157,0.111158$, and 0.111159 with $d=0.1$, from top to bottom. $b$ is set 10 . The straight line which intersects the ordinate at $\approx-0.173$ is a fitting result of $-\delta_{\text {eff }}$ for $p=0.111158$. Inset: Plot of $-\delta_{\text {eff }}$ vs $t^{-0.105}$ at the critical point. The linear extrapolation gives the DP critical exponent.

$\rho_{p}(t)$. In Fig. 5. $-\delta_{\text {eff }}$ obtained using $b=10$ is drawn as a function of $t^{-\chi}$ with $\chi=0.138$ near criticality. From this plot, it is clear that the system with $p=0.111157$ (0.111 159) is in the active (absorbing) phase and the critical point should be $p_{c}=0.111$ 158(1). Although the number of independent runs for both off-critical simulations is only 200, the effective exponents give a clear illustration of the expected behavior in each phase. We fit $-\delta_{\text {eff }}$ for $p=0.111158$ using a linear function to obtain that $\delta \approx 0.173$. Since a fitting result of $\delta$ varies from 0.17 (for $\chi=0.13$ ) to 0.176 (for $\chi=0.15$ ) with $\chi$, we conclude that $\delta=0.173(3)$.

Figure 5 shows that $-\delta_{\text {eff }}$ at criticality becomes an almost straight line in the region $t^{-0.138} \leq 0.25$. This behavior is indeed consistent with the analysis of $\Theta_{p}$. We observed in Fig. 4 that $\Theta_{p}$ for $b=10$ exhibits a nice power-law behavior from $10^{5}$. Recall that $\Theta_{p}$ is calculated using $\rho_{p}$ at $t, t / b$, and $t / b^{2}$. Thus, this numerical observation implies that the leading corrections-toscaling term becomes dominant from $t=10^{3}$. Thus $-\delta_{\text {eff }}$ for $b=10$ should be almost straight from $10^{-4 \chi} \approx 0.25$, as seen in Fig. 5 .

Since the estimated critical exponent 0.173 is close to the DP exponent 0.1595 , it would be an interesting practice which value of $\chi$ can predict the DP critical exponent. By trial and error, we found that $\chi=0.105$ gives the DP critical exponent; see the inset of Fig. 5. Although 0.105 is different from the estimated 0.138 by $25 \%$, it is indeed hard to exclude the possibility of the DP critical scaling. Thus, the analysis of the PCPD with $d=0.1$ alone may not be enough to conclude that the PCPD does not belong to the DP class. To make the estimate 0.173 more convincing, we analyze other cases with different values of $d$.

Before delving into the case with different diffusion probability, we will show that the estimated critical point 


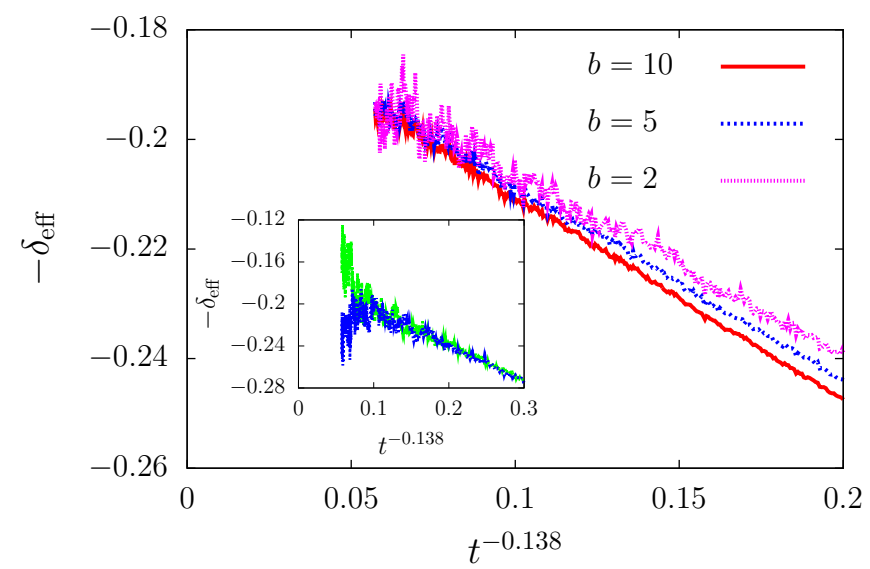

FIG. 6. (Color online) Plots of $-\delta_{\text {eff }}$ vs $t$ at $p=0.111158$ for $b=10,5$, and 2 (from bottom to top). Inset: $-\delta_{\text {eff's for }}$ $b=2$ are plotted against $t^{-0.138}$ at $p=0.111157$ (top) and 0.111159 (bottom).

is rather insensitive to the estimates of $\delta$ and $\chi$. If $\chi$ is small as in the present case, we can approximate $\left(b^{\chi}-1\right) / \ln b \approx \chi+O\left(\chi^{2} \ln b\right)$ as long as $\ln b$ is not so large. Thus, the effective exponent at criticality should be insensitive to $b$. As can be seen in Fig. 6. $-\delta_{\text {eff at }}$ $p=0.111158$ is more or less insensitive to $b$, which strongly supports that the density at $p=0.111158$ exhibits the critical scaling up to the simulated time. Meanwhile, if the system is in the active (absorbing) phase and $t>\left|p-p_{c}\right|^{-\nu_{\|}},-\delta_{\text {eff }}$ at given $t$ should increase (decrease) significantly as $b$ gets smaller. The inset of Fig. [6]depicts $\delta_{\text {eff }}$ at $p=0.111157$ and 0.111159 for $b=2$. By comparing this figure with with Fig. 5], it is easily recognized that $\delta_{\text {eff }}$ at off-criticality is significantly affected by the change of $b$. Although we plotted $-\delta_{\text {eff }}$ vs $t^{-0.138}$ in Fig 6 for convenience, different choice of $\chi$ does not affect the observed behavior of the effective exponents under the change of $b$. Also note that $\delta$ does not play any role in the above discussion. Hence, we conclude that the estimated critical point $p_{c}=0.111158(1)$ is accurate irrespective of whether we are using the right value of $\delta$ and $\chi$.

Now, we will analyze the case of $d=0.5$. Figure 7(a) depicts the CTSFs as functions of $t$ on a double logarithmic scale at $p=0.1524755$ which will turn out to be the critical point. The data are collected from 7000 independent simulation runs and $b=10$ is used. Unlike the case with $d=0.1, \Theta_{r}$ shows a power-law decay $t^{-0.15}$ from about $t=10^{5}$. $\Theta_{p}$ in the short time regime decays faster than $\Theta_{r}$ but after $t=10^{8}, \Theta_{r}$ and $\Theta_{p}$ are almost indistinguishable. Since $\Theta_{r}$ shows a more stable powerlaw behavior than $\Theta_{p}$, we estimate $\chi$ to be 0.15 which is comparable to the above estimate. One can also see that $\Theta_{p}-\Theta_{r}$ decays faster than $t^{-0.15}$.

In Fig. $7(\mathrm{~b}),-\delta_{\text {eff }}$ corresponding to $\rho(t)$ for $b=10$ is drawn against $t^{-0.15}$. The effective exponent for $p=0.152475(0.152476)$ results from 2400 (2500) independent simulation runs. This figure shows that the
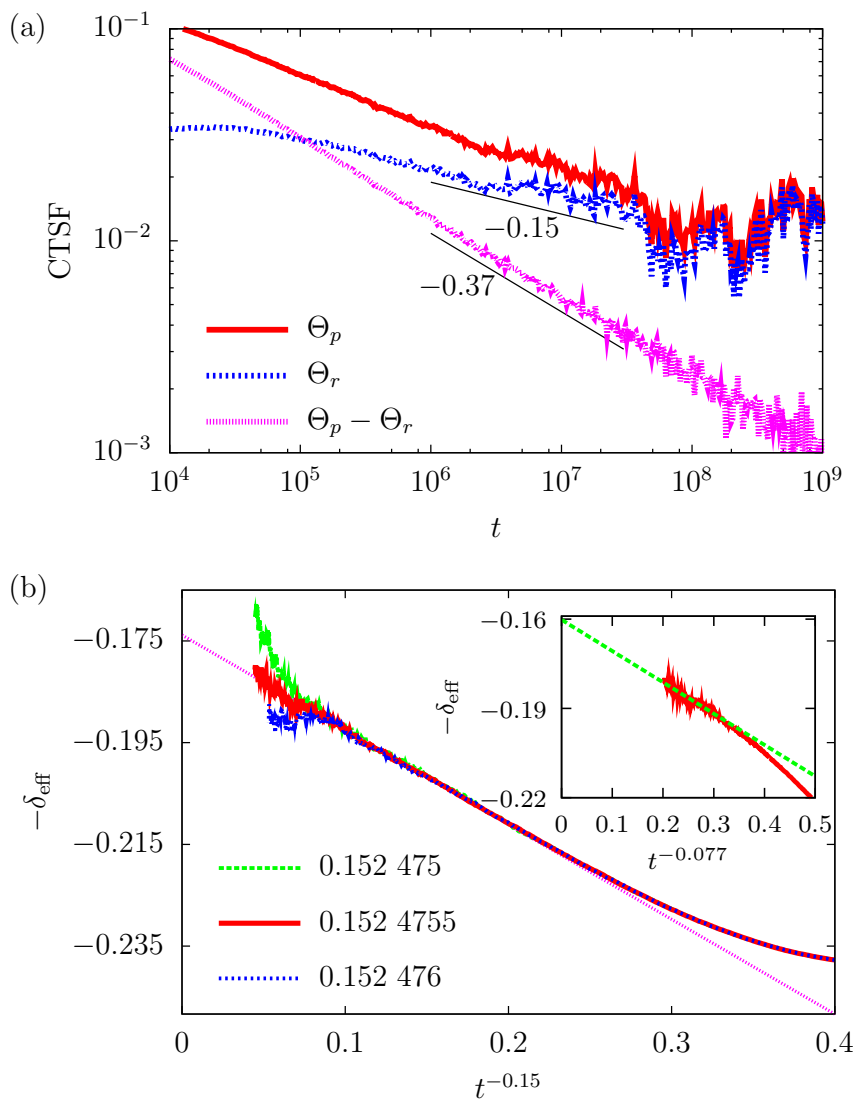

FIG. 7. (Color online) (a) Log-log plots of CTSFs vs $t$ at $p=0.1524755$ and $d=0.5$. Two line segments with slopes -0.15 and -0.37 as indicated in the figure are also drawn for guides to the eyes. (b) Plots of $-\delta_{\text {eff }}$ vs $t$ for $p=0.152475$, 0.1524755 , and 0.152476 with $d=0.5$, from top to bottom. The straight line which meets the ordinate at $\approx-0.174$ is a fitting result of the data for $p=0.1524755$. Inset: Plot of $-\delta_{\text {eff }}$ vs $t^{-0.077}$ at the critical point. The linear extrapolation gives the DP critical exponent.

critical point is $p_{c}=0.1524755(5)$ and a linear fit of $-\delta_{\text {eff for }} p=p_{c}$ gives $\delta \approx 0.174$ which is consistent with the estimate for the case of $d=0.1$. Also note that $-\delta_{\text {eff }}$ is almost straight in the region $t^{-\chi}<10^{-4 \chi} \approx 0.25$, which is consistent with the behavior of $\Theta_{r}$.

We would like to emphasize that the estimate of the critical point is rather insensitive to the accuracy of $\delta$ and $\chi$, so the accuracy of $p_{c}$ is less questionable than the exponents. Using the data of the density at the critical point, we also estimate, by trial and error, the value of $\chi$ which gives the DP exponent. At this time, the desired value of $\chi$ becomes 0.077 which is quite different from the estimated $\chi=0.15$. Furthermore, 0.077 differs by $25 \%$ from the case of $d=0.1$. That is, for our numerical data to be consistent with the DP hypothesis, the LCSE has to vary continuously with $d$ significantly.

Since the case of $d=0.5$ was also studied in Ref. [18] which supports the DP hypothesis, it is worth while to compare our results with those in Ref. [18]. First, the 
(a)

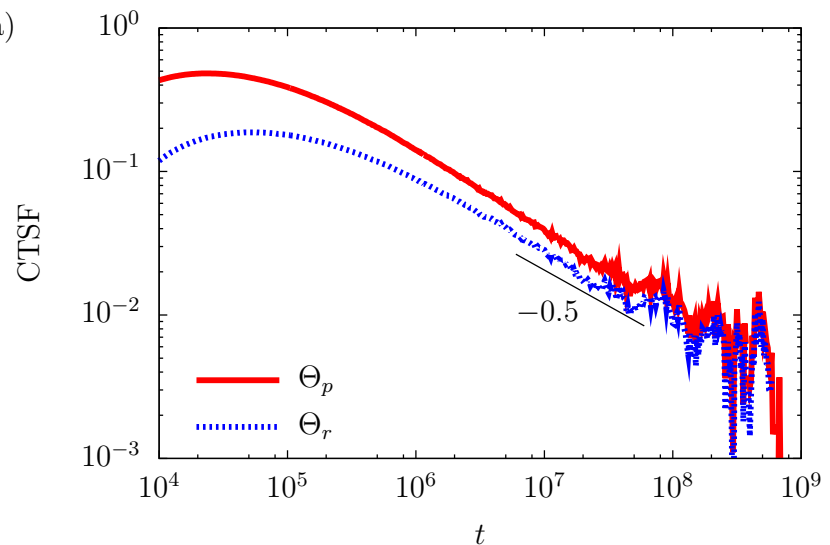

(b)

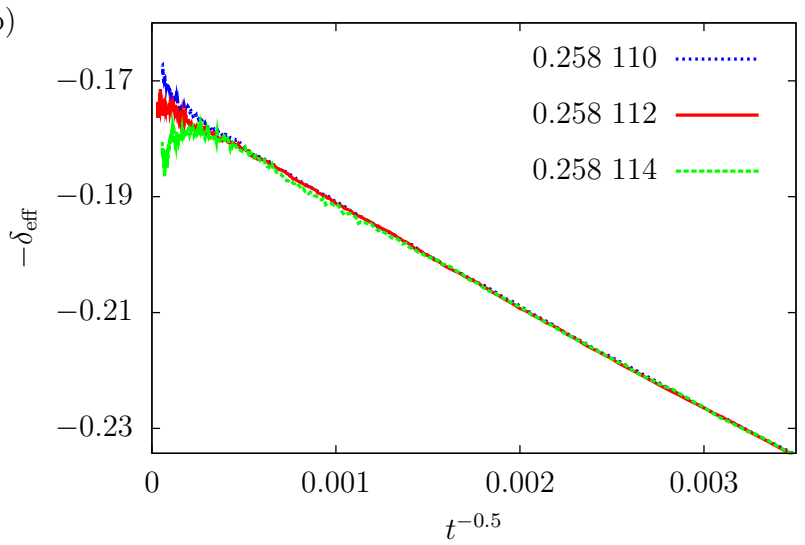

FIG. 8. (Color online) (a) Log-log plots of $\Theta_{p}$ (top curve) and $\Theta_{r}$ (bottom curve) against $t$ at $p=0.258112$ and $d=0.95$. A line segment with slope -0.5 is a guide to the eyes. (b) Plots of $-\delta_{\text {eff }}$ vs $t^{-0.5}$ for $p=0.258110,0.258112$, and 0.258114 with $d=0.95$, from top to bottom.

critical point in this paper is more accurately estimated than in Ref. [18]; see Table I of Ref. [18]. Second, a value close to the DP exponent was obtained from the system at $p=0.152473$ which is actually in the active phase. Because the density in the active phase decays slower than at the critical point, it is not surprising that the estimated value of $\delta$ in Ref. [18] is smaller than 0.173. Interestingly, however, a similar estimate of $\delta$ was attained when the system at $p=0.152476$ was analyzed; see the sixth row of Table 1 in Ref. [18].

The results up to now seem to suggest that the LCSE obtained from the behavior of CTSFs is about 0.15 for the PCPD in general. Quite intriguingly, however, the PCPD with $d=0.95$ has relatively weak corrections to scaling. In Fig. 8(a), the CTSFs for $b=10$ at the critical point of the case with $d=0.95$ are depicted as functions of $t$ on a double-logarithmic scale. Note that $\Theta_{r}$ in this figure is the middle curve in Fig. 1. Unlike the previous two cases, $\Theta_{r}$ decays as $t^{-0.5}$ rather than $t^{-0.15}$. Note that the power-law behavior of $\Theta_{r}$ is observed from $t=10^{6}$ which means the LCSE in $\rho(t)$ becomes dominant from $t=10^{4}$.

Using this LCSE, we depict $-\delta_{\text {eff }}$ calculated from $\rho(t)$

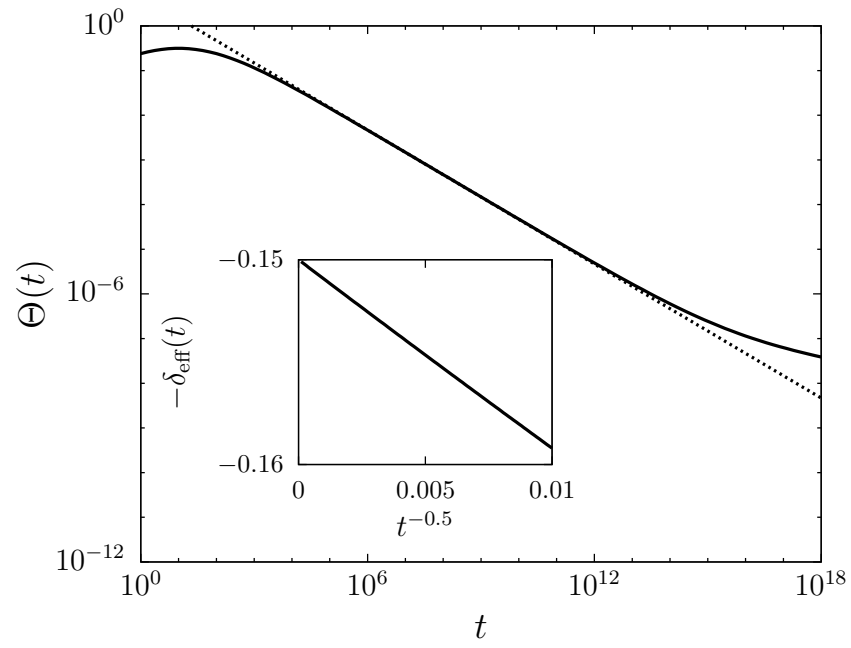

FIG. 9. Log-log plot of $\Theta$ vs $t$ of the toy equation. The straight line with slope -0.5 is a guide to the eyes. Up to $t=10^{12}, \Theta(t)$ shows a nice power-law behavior of $t^{-0.5}$. Inset: Plot of $-\delta_{\text {eff }}$ vs $t^{-0.5}$. Although the true leading correctionsto-scaling term of Eq. (16) is $t^{-0.15},-\delta_{\text {eff drawn as a function }}$ of $t^{-0.5}$ gives the exact leading term.

with $b=10$ as a function of $t^{-0.5}$ in Fig. 8 (b) which again shows a typical behavior of $-\delta_{\text {eff }}$ near criticality. From the behavior of $-\delta_{\text {eff }}$ we estimate $p_{c}=0.258112(2)$. A linear fit of $-\delta_{\text {eff }}$ for $p=0.258112$ suggests $\delta \approx 0.173$, which is again consistent with the estimates from the cases of different $d$ 's. Since the LCSE is dominant from $10^{4}$, the effective exponent should be a straight line for $t^{-\chi}<10^{-5 \chi} \approx 0.003$, as seen in Fig. 8 (b).

We also investigated which value of $\chi$ can give the DP exponent for the high diffusion case. Unlike the low diffusion cases, however, the DP exponent was hardly observed by varying $\chi$, which seems to imply that the critical behavior of the PCPD with $d=0.95$ cannot be consistent with the DP hypothesis.

Since $\chi$ needs not be universal, appearing dependence of $\chi$ on $d$ is not contradictory to our common sense formed by the renormalization group (RG) theory. Still, what kind of mathematical structure is behind the change of $\chi$ with $d$ is an interesting question.

We think there are two possible scenarios. The first obvious scenario is that $\chi$ is a continuous function of $d$. Without a fixed line in the RG sense, however, it is hard to expect such a continuously varying exponent albeit non-universal, so this scenario does not look plausible. The second one is that the correction term $t^{-0.15}$ is actually present even in the case with $d=0.95$ but the coefficient is very small.

The implication of the second scenario can be clearly stated by the following toy equation

$$
\rho(t)=t^{-0.15}\left(1+10^{-4} t^{-0.15}+t^{-0.5}\right) .
$$

In this toy example, the leading corrections-to-scaling term becomes dominant only when $t \gg 2.6 \times 10^{11}$ and before this time $t^{-0.5}$ plays the role of the leading 
TABLE I. Critical points of the PCPD for various $d$. The numbers in parentheses indicate uncertainty of the last digits.

\begin{tabular}{ll}
\hline \hline$d$ & $p_{c}$ \\
\hline 0 & $0.0770905(5)^{\mathrm{a}}$ \\
0.001 & $0.1019(1)^{\mathrm{b}}$ \\
0.005 & $0.1023(1)^{\mathrm{b}}$ \\
0.01 & $0.1028(1)^{\mathrm{b}}$ \\
0.02 & $0.1038(1)^{\mathrm{b}}$ \\
0.05 & $0.1066(1)^{\mathrm{b}}$ \\
0.1 & $0.111158(1)$ \\
$\frac{1}{3}$ & $0.133519(3)^{\mathrm{c}}$ \\
0.5 & $0.1524755(5)$ \\
0.9 & $0.2334(1)^{\mathrm{b}}$ \\
0.95 & $0.258112(2)$ \\
0.99 & $0.2968(1)^{\mathrm{b}}$ \\
\hline \hline
\end{tabular}

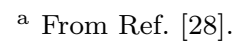

b detailed analysis not shown in the paper.

${ }^{c}$ From Ref. [14].

${ }^{d}$ Mean field critical point. See Sec. IVB

corrections-to-scaling term. Thus, $\Theta(t)$ is well described by $t^{-0.5}$ and the corresponding effective exponent drawn as a function of $t^{-0.5}$ rather than the true asymptotic behavior $t^{-0.15}$ gives the correct value of 0.15 ; see Fig. 9 , We think the second scenario is plausible, but more investigation is necessary to fully understand how the mathematical structure of the corrections to scaling changes with $d$. This also can be a theoretical challenge of the PCPD. In any case, $\Theta(t)$ is a useful tool to find the correct value of the critical exponent, although it may not predict the true LCSE as in the toy example.

To conclude this section, we found that the critical decay exponent of the PCPD is robust against $d$ with value $\delta=0.173(3)$. Although this value differs from the DP value only by $8 \%$, the consistent estimate for a wide range of $d$ supports the non-DP hypothesis. In particular, the DP hypothesis is not consistent with the case with $d=0.95$ up to the simulation time in this work $\left(t=10^{9}\right)$. Since the corrections-to-scaling for the high diffusion case is much weaker than those for the low diffusion case, it seems promising to find other exponents accurately by investigating the PCPD for large $d$.

\section{CROSSOVER}

To get a hint to the crossover behavior occurring at two limiting cases $d=0$ and $d \rightarrow 1$, we found critical points for a wide range of $d$, which are summarized in Table [ Because the estimate of the critical points within error $10^{-4}$ is relatively easy with the present computing power, we just present the resulting critical points without showing the details.

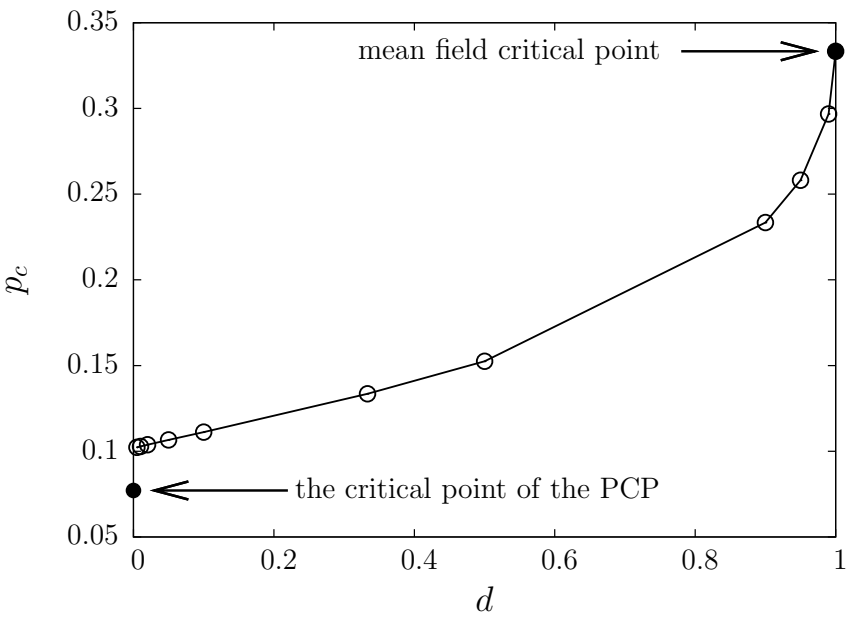

FIG. 10. Plot of $p_{c}$ vs $d$ for the PCPD. The critical point of the PCP without diffusion and the mean field critical point are indicated by respective arrows.

The phase boundary of the PCPD in the $d-p$ plane shown in Fig. 10 has two salient features at the two end points, $d=0$ and $d=1$. The phase boundary is discontinuous at $d=0$ and the phase boundary approaches the mean field critical point with infinite slope as $d \rightarrow 1$. Each singular behavior signifies a crossover; crossover from the PCP to the PCPD at $d=0$ and that from the mean field PCPD to the one dimensional PCPD at $d=1$. In this section, we will investigate these two kinds of crossover behavior one by one and find the corresponding crossover exponents.

\section{A. From the PCP to the PCPD}

The discontinuity of the phase boundary at $d=0$ can be understood as follows. In this discussion, all exponents are of the DP class. Consider the system at $p=p_{c}(0)+\Delta p$ with $0<\Delta p \ll p_{c}(0)$, where $p_{c}(0)$ is the critical point of the PCP. If $d=0$, the system is in the absorbing phase and the pair density decays exponentially if time $t$ exceeds the relaxation time $\xi_{t} \sim \Delta p^{-\nu_{\|}}$. On the other hand, the particle density should approach a certain nonzero value $\rho_{s}(p)$. If $0<d \xi_{t} \ll \rho_{s}(p)^{-2}$, it is unlikely for isolated particles to meet each other purely by diffusion when $t$ is smaller than $\xi_{t}$. Hence, effectively, the initial PCP dynamics is almost decoupled with diffusion before $t=\xi_{t}$ and only when $t$ exceeds $\xi_{t}$ pairs formed by diffusion can appear. Since we are considering an infinite system, the pair density is nonzero for any finite $t$ although it can be extremely small. As soon as pairs appear by diffusion, the so-called defect dynamics of the PCP begins. Since the probability that two consecutive sites are occupied by diffusion is roughly $\rho_{s}(p)^{2}$, the mean distance between two pairs formed by diffusion should be $1 / \rho_{s}(p)^{2}$. Let $P\left(\xi_{x}\right)$ be the probability that the defect dynamics continues until the cluster size be- 


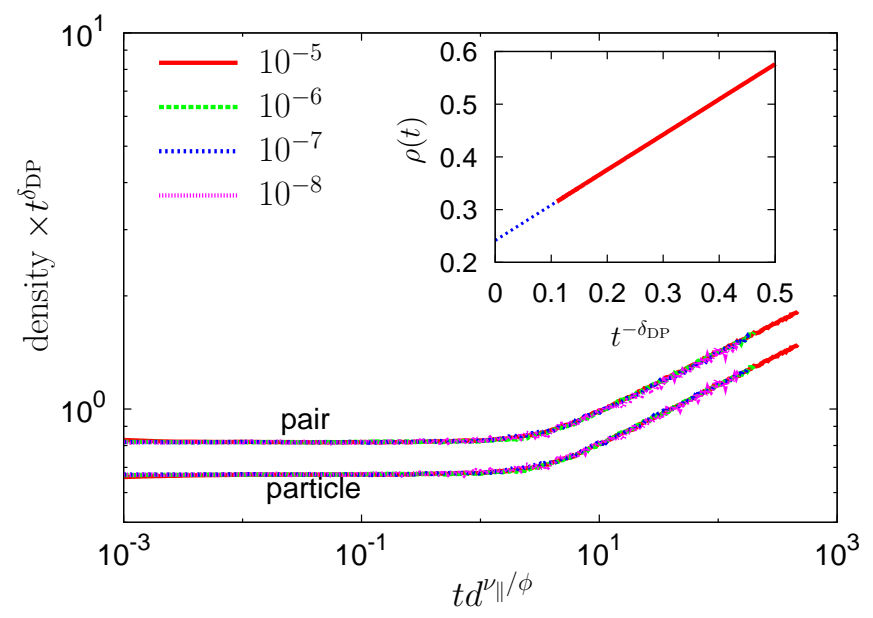

FIG. 11. (Color online) Log-log plots of $\rho_{p} t^{\delta_{\mathrm{DP}}}$ vs $t d^{\nu_{\|} / \phi}$ (top curves) and $\left(\rho-\rho_{s}\right) t^{\delta_{\mathrm{DP}}}$ vs $t d^{\nu_{\|} / \phi}$ (bottom curves) for $d=10^{-5}, 10^{-6}, 10^{-7}$, and $10^{-8} \cdot \rho_{s}$ is the steady state density of particles for $d=0$, which is estimated as $\approx 0.2414$. $\delta_{\mathrm{DP}} \simeq$ 0.1595 and $\nu_{\|} \simeq 1.732$ are the critical exponents of the DP. In the scaling collapse, we use $\phi=2.6$. Inset: Plot of $\rho(t)$ vs $t^{-\delta_{\mathrm{DP}}}$ at the PCP critical point. The extrapolation gives $\rho_{s}=0.2414(3)$.

comes $\xi_{x} \sim \Delta p^{-\nu_{\perp}} . P\left(\xi_{x}\right)$ should be the same order as the survival probability that the defect dynamics continues until $\xi_{t}$, which for small $\Delta p$ becomes $\xi_{t}^{-\beta / \nu_{\|}} \sim \Delta p^{\beta}$. This is because the scaling form of the survival probability is $t^{-\beta / \nu_{\|}} f\left(t / \xi_{t}\right)$, where $f(x)$ is a scaling function with finite value of $f(1)$. Then the mean distance between the starting points of two successfully spreading clusters should be $\sim 1 /\left[\rho_{s}(p)^{2} P\left(\xi_{x}\right)\right]$. If $\xi_{x} \gg 1 /\left[\rho_{s}(p)^{2} P\left(\xi_{x}\right)\right]$ or $\rho_{s}(p)^{2} \xi_{x} P\left(\xi_{x}\right) \gg 1$, a merger of two spreading clusters into a single cluster happens frequently and the system should survive indefinitely. Hence, the condition that the system dynamics continue indefinitely by any small but finite $d$ is $\rho_{s}(p)^{2} \xi_{x} P\left(\xi_{x}\right) \gg 1$ or $\rho_{s}(p)^{2}(\Delta p)^{-\nu_{\perp}+\beta} \gg 1$. Since $\nu_{\perp}>\beta$, there should be a finite range of $\Delta p$ which satisfies the above criterion. Thus, the phase boundary should be discontinuous at $d=0$. Using $\rho_{s}(p)$ at the critical point of the PCP (see the inset of Fig. 11) and the $\mathrm{DP}$ exponents $\beta \approx 0.27, \nu_{\perp} \approx 1.09$, the validity of the above criterion gives $\Delta p<0.03$ which is comparable to the numerical result.

Due to the discontinuity, the phase boundary does not give any information about the crossover exponent $\phi$. Rather, we find $\phi$ by data collapse using the following scaling ansatz,

$$
\begin{gathered}
\rho(p, d ; t)=\rho_{s}(p)+t^{-\delta_{\mathrm{DP}}} \Psi_{\rho}\left(t\left|p-p_{c}(0)\right|^{\nu_{\|}}, t d^{\nu_{\|} / \phi}\right) \\
\rho_{p}(p, d ; t)=t^{-\delta_{\mathrm{DP}}} \Psi_{p}\left(t\left|p-p_{c}(0)\right|^{\nu_{\|}}, t d^{\nu_{\|} / \phi}\right)
\end{gathered}
$$

where $\rho_{s}(p)$ is the steady state particle density at $d=0$, $\phi$ is the crossover exponent, $\Psi_{\rho}, \Psi_{p}$ are scaling functions, and $\delta_{\mathrm{DP}} \approx 0.1595$ and $\nu_{\|} \approx 1.732$ are the critical exponents of the DP class. We observe the best collapse when we use $\phi=2.6$ as shown in Fig. 11. Thus, we conclude $\phi=2.6(1)$. Note that this crossover exponent is different from that of the crossover from the DP class with infinitely many absorbing states to the DP class with finite number of absorbing states [28].

The existence of the nontrivial crossover behavior at $d=0$ has nothing to do with the change of universality classes. In fact, this crossover originates from the drastic decrease of the volume of absorbing states in the configuration space 28]. However, the discontinuity at $d=0$ in the phase boundary raises a criticism on the Hinrichsen's argument explained in Sec. [I. This criticism starts from numerical observation that diffusion makes the system more active as the system at $p=p_{c}(0)$ with nonzero $d$ is in the active phase. Now consider the system at $p=p_{c}(0)$ and $0<d \ll 1$. In this case, clusters spreads even faster than the critical spreading in the long time limit. Repeating Hinrichsen's argument, one can conclude that diffusion of isolated particles is negligible in the long time limit and, in turn, isolated particles can join a cluster by the spreading of clusters not by their own diffusion, which is the crucial feature of immobile isolated particles in the PCP. If this were the case, the critical point should approach $p_{c}(0)$ as $d \rightarrow 0$ and the phase boundary should be continuous at $d=0$ just as the inhibitory route in Ref. 28]. Obviously, this is contradictory to the numerical result. Also note that the origin of the discontinuity is the active role of isolated particles, which is completely neglected in the Hinrichsen's argument. In other words, one cannot deduce a right conclusion by simply comparing the speed of spreading clusters with that of diffusion. Hence, we cannot neglect the effect of pure diffusion and there should be a strong correlation between diffusion and the critical cluster spreading, which can mediate the change of the universality class.

\section{B. From the mean field PCPD to the PCPD}

The mean field equation for the PCPD is obtained by setting $\rho_{p}(t)=\rho(t)^{2}$ and $\rho_{t}(t)=\rho(t)^{3}$ in Eq. (3), which gives

$$
\frac{d \rho}{d \tau}=(1-3 p) \rho^{2}-(1-p) \rho^{3}
$$

where $\tau=(1-d) t$. The critical point of the mean field theory is $p_{0}=\frac{1}{3}$ at which the density decays as

$$
\rho_{c}(\tau)=\frac{\rho_{0}}{\left(1+4 \rho_{0}^{2} \tau / 3\right)^{1 / 2}} \sim \tau^{-1 / 2},
$$

where $\rho_{0}$ is the initial density (we will set $\rho_{0}=1$ ). When $3\left|p_{0}-p\right| \rho_{c}^{2} \ll(1-p) \rho_{c}^{3}, \rho(t)$ is indistinguishable from $\rho_{c}(t)$ and clear deviation from Eq. (19) is observable when $3\left|p_{0}-p\right| \rho_{c}^{2} \gg(1-p) \rho_{c}^{3}$, or $\left|p-p_{0}\right| \tau^{-1} \gg \tau^{-3 / 2}$. In other words, the critical density decay is observable when $\tau \ll\left|p-p_{0}\right|^{-2}$ and the off-critical behavior dominates when $\tau \gg\left|p-p_{0}\right|^{-2}$. Hence, the critical exponent $\nu_{\|}$for the mean field theory is 2 . 
(a)

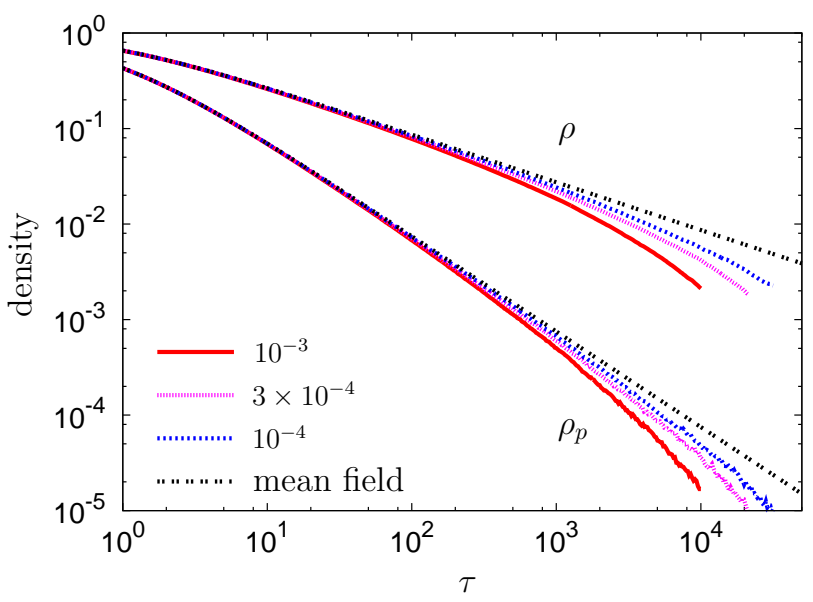

(b)

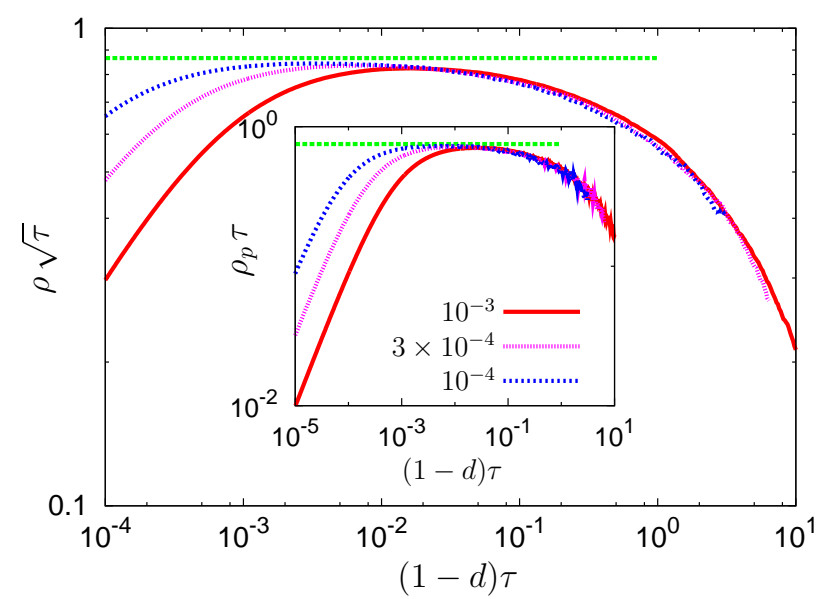

FIG. 12. (Color online) (a) Plots of particle density $\rho$ and pair density $\rho_{p}$ as functions of $\tau=(1-d) t$ for $1-d=10^{-3}$, $3 \times 10^{-4}, 10^{-4}$, and the mean field theory (bottom to top for each group). (b) Scaling collapse plot of $\rho \sqrt{\tau}$ vs $(1-d) \tau$ at $p=p_{0}$ for same $d$ 's in (a). For comparison, $\Phi_{\rho}(0,0)=\sqrt{3} / 2$, is drawn as a line segment. Inset: Scaling collapse plots of $\rho_{p} \tau$ vs $(1-d) \tau$ for the same values of $d$ For comparison $\Phi_{p}(0,0)=$ $\frac{3}{4}$ is drawn as a line segment.

The relation between the mean field equation and the PCPD under $d \rightarrow 1$ limit can be understood as follows (similar argument is also found in Ref. [29]). Under the limit $d \rightarrow 1$ with $\tau$ kept finite, any correlation generated by reaction dynamics will be removed by diffusion immediately. Since the mean field theory assumes no correlation for all time $\tau$, Eq. (18) becomes exact in this limit. Even for finite $1-d$, the mean field equation is an accurate approximation if the relaxation time of diffusion of a randomly chosen region is much smaller than the time between two consecutive reaction dynamics in the same region.

To find the criterion for the validity of the mean field theory for small but finite $1-d$, consider the mean field dynamics at $p=p_{0}$. According to the mean field solu- tion, the mean distance $\ell(\tau)$ at time $\tau$ between two nearest particles is $\ell(\tau)=\rho^{-1} \sim \tau^{1 / 2}$. Now consider a region of size $O(\ell(\tau))$ at time $\tau$ and assume that two consecutive reaction events occur at $\tau$ and $\tau+\Delta \tau$ in this region. We also assume that during $\Delta \tau$, this region is not correlated with outside of this region. Since the number of particles is finite in this region, $\Delta \tau$ should be $O(1)$; recall that $\tau$ is rescaled time. Since the diffusion constant in rescaled time $\tau$ is $d /(1-d) \approx 1 /(1-d)$, the relaxation time of diffusion of this region is $O\left(\ell(\tau)^{2}\right)(1-d) \sim(1-d) \tau$. If $(1-d) \tau \ll \Delta \tau \sim 1$, two consecutive reaction events are uncorrelated and the mean field theory becomes accurate. On the other hand, if $(1-d) \tau \gg 1$, two consecutive reaction events become correlated and the mean field theory fail to describe the system correctly. Hence, the crossover from the mean field PCPD to the PCPD occurs when $\tau \sim(1-d)^{-1}$ and $(1-d) \tau$ becomes a proper scaling parameter.

According to the above argument, the particle density in the asymptotic regime should take the scaling form

$$
\rho(p, d, \tau)=\tau^{-1 / 2} \Phi_{\rho}\left(\tau\left|p-p_{0}\right|^{2}, \tau(1-d)\right),
$$

where $\tau=(1-d) t$ as above and $\Phi_{\rho}$ is a scaling function. We also conjecture the scaling form of the pair density as

$$
\rho_{p}(p, d, \tau)=\tau^{-1} \Phi_{p}\left(\tau\left|p-p_{0}\right|^{2}, \tau(1-d)\right),
$$

where $\Phi_{p}$ is another scaling function. From the mean field theory, $\Phi_{\rho}(0,0)=\sqrt{3} / 2$ and $\Phi_{p}(0,0)=3 / 4$. Hence, if $1-d \ll 1$ and $p=p_{0}$, plots of $t^{1 / 2} \rho\left(p_{0}, d, \tau\right)$ against $(1-d) \tau$ should collapse onto a single curve for sufficiently large $(1-d) \tau$. Furthermore, the phase boundary for $1-d \ll 1$ should approach the mean field critical point as

$$
\left|p_{c}(d)-p_{0}\right| \sim(1-d)^{1 / 2},
$$

where $p_{c}(d)$ is the critical point of the PCPD for given $d<1$. Hence, the crossover exponent is $\phi=2$.

To confirm the above argument, we simulated the PCPD for $1-d=10^{-3}, 3 \times 10^{-4}$, and $10^{-4}$ at $p=\frac{1}{3}$ with the system size $L=2^{22}$. In Fig. 12(a), we depict $\rho(\tau)$ vs $\tau$ and $\rho_{p}(\tau)$ vs $\tau$ on a double-logarithmic scale. For comparison, the mean field solution is also depicted. As argued, the regime where mean field theory is accurate becomes larger as $d$ gets closer to 1. In Fig. 12(b), one can see a scaling collapse plot of $\rho \sqrt{\tau}$ vs $(1-d) \tau$ as well as $\rho_{p} \tau$ vs $(1-d) \tau$, which affirms that the crossover exponent is 2 .

\section{SUMMARY}

To sum up, we numerically studied the critical density decay of the pair contact process with diffusion (PCPD) and estimated the critical decay exponent by investigating effective exponents after finding corrections to scaling 
for various diffusion strength. For small diffusion probability $(d \leq 0.5)$, we found that the corrections-to-scaling term asymptotically behaves as $t^{-0.15}$ and for large diffusion probability $(d=0.95)$ the corrections-to-scaling term decays as $t^{-0.5}$ which is weaker than that of the case with small $d$. All the same, the analysis of the effective exponents for any $d$ with the corresponding correctionsto-scaling term showed that the critical decay exponent is $\delta=0.173(3)$. Although this value is quite close to that of the directed percolation (DP) universality class which is 0.1595, the systematic deviation of $\delta$ for the PCPD from the DP value for any $d$ suggests that the PCPD does not belong to the DP class and forms an independent universality class.

We also studied the crossover from the pair contact process (PCP) without diffusion to the PCPD which occurs around $d=0$ and from the mean field theory (MFT) to the PCPD which happens around $d=1$. We found that the crossover at $d=0$ is characterized by the discontinuity of the phase boundary and that the crossover exponent is $\phi=2.6(1)$. We showed that applying the Hinrichsen's argument [22] which supports the DP hypothesis to this crossover leads to a contradictory conclu- sion to the discontinuity of the phase boundary at $d=0$. The crossover from the MFT to the PCPD, occurring at $d=1$, is described by the crossover exponent $\phi=2$, which was argued to be exact.

\section{ACKNOWLEDGMENTS}

This work was supported by the Basic Science Research Program through the National Research Foundation of Korea (NRF) funded by the Ministry of Education, Science and Technology (Grant No. 2011-0014680); and by the Catholic University of Korea, Research Fund, 2013. The author acknowledges the hospitality of the Institut für Theoretische Physik, Universität zu Köln, Germany and support under a German Research Foundation (DFG) grant within SFB 680 Molecular Basis of Evolutionary Innovations during the final stage of this work. The computation was supported by Universität zu Köln. The author also would like to thank the APCTP for its hospitality.
[1] P. Grassberger, Z. Phys. B 47, 365 (1982).

[2] M. J. Howard and U. C. Täuber, J. Phys. A 30, 7721 (1997).

[3] G. Ódor, Phys. Rev. E 62, R3027 (2000).

[4] E. Carlon, M. Henkel, and U. Schollwöck, Phys. Rev. E 63, 036101 (2001).

[5] H. Hinrichsen, Phys. Rev. E 63, 036102 (2001).

[6] K. Park and I.-M. Kim, Phys. Rev. E 66, 027106 (2002).

[7] G. T. Barkema and E. Carlon, Phys. Rev. E 68, 036113 (2003).

[8] J. Kockelkoren and H. Chaté, Phys. Rev. Lett. 90, 125701 (2003).

[9] H.-K. Janssen, F. van Wijland, O. Deloubriere, and U. C. Täuber, Phys. Rev. E 70, 056114 (2004).

[10] J. D. Noh and H. Park, Phys. Rev. E 69, 016122 (2004).

[11] S.-C. Park and H. Park, Phys. Rev. Lett. 94, 065701 (2005).

[12] S.-C. Park and H. Park, Phys. Rev. E 71, 016137 (2005).

[13] M. M. de Oliveira and R. Dickman, Phys. Rev. E 74, 011124 (2006).
[14] S.-C. Park and H. Park, Phys. Rev. E 73, 025105 (2006).

[15] S. Kwon and Y. Kim, Phys. Rev. E 75, 042103 (2007).

[16] F. Smallenburg and G. T. Barkema, Phys. Rev. E 78, 031129 (2008).

[17] S.-C. Park and H. Park, Phys. Rev. E 79, 051130 (2009).

[18] R. D. Schram and G. T. Barkema, J. Stat. Mech.:Theory Exp. (2012), P03009.

[19] D. Gredat, H. Chaté, B. Delamotte, and I. Dornic, Phys. Rev. E 89, 010102 (2014).

[20] G. Ódor, M. C. Marques, and M. A. Santos, Phys. Rev. E 65, 056113 (2002).

[21] M. Henkel and H. Hinrichsen, J. Phys. A 37, R117 (2004).

[22] H. Hinrichsen, Physica A 361, 457 (2006).

[23] S.-C. Park and H. Park, Eur. Phys. J. B 64, 415 (2008).

[24] H.-K. Janssen, Z. Phys. B 42, 151 (1981).

[25] S.-C. Park, J. Korean Phys. Soc. 62, 469 (2013).

[26] I. Jensen, Phys. Rev. Lett. 70, 1465 (1993).

[27] G. Ódor, Phys. Rev. E 67, 016111 (2003).

[28] S.-C. Park and H. Park, Phys. Rev. E 76, 051123 (2007).

[29] S.-C. Park, Phys. Rev. E 80, 061103 (2009). 